\title{
Unsteady MHD flow and heat transfer over a stretching/shrinking permeable sheet with ohmic heating Zaileha Md Ali ${ }^{1}$, Nurulnabila Jamaruddin ${ }^{1}$, Siti Khuzaimah Soid ${ }^{1}$, Nur Aziean Mohd Idris ${ }^{1}$, Md Nizam Udin ${ }^{2}$, Nur Zahidah Ismail ${ }^{1}$
}

\author{
${ }^{1}$ Faculty of Computer and Mathematical Sciences, \\ Universiti Teknologi MARA Shah Alam Selangor, Malaysia \\ ${ }^{2}$ Faculty of Computer and Mathematical Sciences, \\ Universiti Teknologi MARA, Seremban, Negeri Sembilan, Malaysia
}

*Corresponding author: zaileha@tmsk.uitm.edu.my

Received: 09 September 2019; Accepted: 19 December 2019; Published: 23 December 2019

\begin{abstract}
The problem of an unsteady magnetohydrodynamics (MHD) and heat transfer over a stretching/shrinking permeable sheet with ohmic heating is studied in this paper. Using a nonlinear partial differential equations system, the physical problem is modeled and transformed into ordinary differential equations using similarity transformation. Along with the boundary conditions, the equations are solved numerically using bvp4c in the MATLAB software and a comparison with previous literature shows an excellent agreement. The effect of suction parameter, unsteady parameter and magnetic parameter in porous plate are discussed. The results show that the velocity profile and skin friction coefficient increases when suction parameter increases. Furthermore, the increase in magnetic and unsteady parameters resulted in the increase in the velocity profile and the skin friction coefficient respectively.
\end{abstract}

Keyword : Magnetohydrodynamics, MATLAB softwere

\section{INTRODUCTION}

Magnetohydrodynamics or MHD is the magnetic properties of electrically conducting fluids dynamics such as saltwater, plasmas and liquid metals. In metal-working processes and modern metallurgical, the study of MHD flow of an electrically conducting fluid is significantly important. Magnetic field applied in heat transfer and regulating momentum in the boundary layer of distinct fluids over a stretching sheet causes the streamline steeper that produce a thinning boundary layer [1]. Stretching consists of a large finite structure to its infinite version about a surface while shrinking describes an infinite structure to its finite approximation about a surface [2]. The useful applications for the study of MHD flow over a stretching/shrinking sheet in industrial processes are manufacturing of glass sheets, polymer processing, textile industries and paper production [3]

Ohmic heating is indicated as Joule heating, electro-conductive heating, direct electrical resistance heating or electro-heating [4]. A study of an unsteady MHD stagnation point flow with ohmic heating and viscous dissipation over a stretching/shrinking sheet was conducted by [5]. They found that dual solutions exist for the shrinking case, while there is a unique solution for the stretching case. It shows that the significant effect on the flow and the heat transfer rate affected by the presence of unsteadiness and magnetic parameters [5]. Ohmic heating has received special interest due to its potential benefits such as in food industry, waste treatment, water distillation and chemical processing $[6,7,8,9]$. However, there is a limited study done regarding the heat transfer and an unsteady MHD flow over a stretching/shrinking sheet especially involvement of permeable plate due to its huge implementations in engineering, geophysics, and thermal sciences [10]. 
This paper extends and investigates the study conducted by [5] by considering the permeable sheet on the effect of suction in the heat transfer and an unsteady MHD flow with ohmic heating over a stretching/shrinking permeable sheet.

\section{MATHEMATICAL FORMULATION}

Consider a viscous electrically conducting fluid over a permeable stretching/shrinking sheet on heat transfer and an unsteady stagnation point flow. The variable $x$ measures along the surface of the sheet while the variable $y$ is normal to it. The permeable stretching or shrinking sheet velocity is $u_{w}=\lambda u_{w}$ where $\lambda$ is a constant with $\lambda>0$ refers to a stretching sheet and $\lambda<0$ points to a shrinking sheet, $u_{e}$ is the velocity of inviscid flow, $T_{w}$ is the sheet temperature, $T_{\infty}$ is the ambient temperature and $B$ is the transverse magnetic field strength (Soid et al.,2017).

$$
\begin{aligned}
& \frac{\partial u}{\partial x}+\frac{\partial v}{\partial y}=0 \\
& \frac{\partial u}{\partial t}+u \frac{\partial u}{\partial x}+v \frac{\partial u}{\partial y}=\frac{\partial u_{e}}{\partial t}+u_{e} \frac{\partial u_{e}}{\partial x}+v \frac{\partial^{2} u}{\partial y^{2}}+\frac{\sigma B^{2}}{\rho}\left(u_{e}-u\right) \\
& \frac{\partial T}{\partial t}+u \frac{\partial T}{\partial x}+v \frac{\partial T}{\partial y}=\alpha \frac{\partial^{2} T}{\partial y^{2}}+\frac{v}{C_{p}}\left(\frac{\partial u}{\partial y}\right)^{2}+\frac{\sigma B^{2}}{\rho C_{p}}\left(u_{e}-u\right)^{2}
\end{aligned}
$$

along with the initial and boundary

$$
\begin{aligned}
& t<0: \quad u=v=0, \quad T=T_{\infty} \text { for any } x, y \\
& t \geq 0: \quad u=\lambda u_{w}(x, t), \quad v=V_{0}, \quad T=T_{w}(x, t) \text { at } y=0 \\
& u \rightarrow u_{e}(x, t), \quad T \rightarrow T_{\infty} \text { as } \quad y \rightarrow \infty
\end{aligned}
$$

where $u$ and $v$ are the velocity for the $x$ - and $y$-axes, $v$ is the fluid kinematic viscosity, $\sigma$ is the fluid electrical conductivity, $\rho$ is the density, $T$ is the fluid temperature, $t$ is the time, $\alpha$ is the fluid thermal diffusivity, $C_{p}$ is the specific heat at constant pressure and $V_{0}$ is the surface mass flux. It is assumed that $u_{w}(x, t)$ , $u_{e}(x, t), T_{w}(x, t)$ and $B^{2}(t)$ are as in Equation (5), hence Equations (1) to (4) admit to similarity solutions.

$$
u_{w}(x, t)=\frac{a x}{1-\beta t}, \quad u_{e}(x, t)=\frac{a x}{1-\beta t}, T_{w}(x, t)=T_{\infty}+\frac{b x^{2}}{(1-\beta t)^{2}}, \quad B^{2}(t)=\frac{B_{0}^{2}}{1-\beta t}
$$

where $a>0$ and $b$ are constant, $\beta$ is a constant denoted with the dimension (time) $)^{-1}$ and $B_{0}$ is the constant of applied magnetic field and the variables being considered are

$\psi=\left(\frac{a v}{1-\beta t}\right)^{1 / 2} x f(\eta), \quad \eta=\left(\frac{a}{v(1-\beta t)}\right)^{1 / 2} y, \quad \theta=\frac{T-T_{\infty}}{T_{w}-T_{\infty}}$

where $\psi$ is defined as $u=\partial \psi / \partial y$ and $v=-\partial \psi / \partial x, \eta$ is the dimensionless similarity variable and $\theta$ is the dimensionless temperature. Substituting Equation (6) into Equations (1) to (4) leads to the ordinary differential equations: 


$$
\begin{aligned}
& f^{\prime \prime \prime}+f f^{\prime \prime}+1-f^{\prime 2}-A\left(f^{\prime}-1+\frac{\eta}{2} f^{\prime \prime}\right)+M\left(1-f^{\prime}\right)=0 \\
& \frac{1}{\operatorname{Pr}} \theta^{\prime \prime}+f \theta^{\prime}-2 f^{\prime} \theta-A\left(2 \theta+\frac{1}{2} \eta \theta^{\prime}\right)+E c\left[M\left(1-f^{\prime}\right)^{2}+f^{\prime \prime 2}\right]=0
\end{aligned}
$$

subject to the boundary conditions as

$f(0)=S, \quad f^{\prime}(0)=\lambda, \quad \theta(0)=1$

$f^{\prime}(\eta) \rightarrow 1, \quad \theta(\eta) \rightarrow 0 \quad$ as $\eta \rightarrow \infty$

where primes represent differentiation with respect to $\eta$ and $S>0$ is the suction parameter while $S<0$ is the injection parameter. This paper focuses on the effect of suction parameter. Parameter $A$ represents the unsteadiness, $M$ is the constant magnetic parameter, $E c$ is the Eckert number and $\operatorname{Pr}$ is the Prandtl number which are defined as

$$
A=\frac{\beta}{a} \quad, M=\frac{\sigma B_{0}{ }^{2}}{a \rho}, E c=\frac{u_{w}{ }^{2}}{C_{p}\left(T_{w}-T_{\infty}\right)}, \operatorname{Pr}=\frac{u}{\alpha} .
$$

Using Equation (6), thus obtains

$\operatorname{Re}_{x}^{1 / 2} C_{f}=f^{\prime \prime}(0), \quad \operatorname{Re}_{x}^{-1 / 2} N u_{x}=-\theta^{\prime}(0) \quad$ where $\operatorname{Re}_{x}^{1 / 2}=\left(\frac{a}{v}\right)^{1 / 2} x$

\section{RESULTS AND DISCUSSION}

Table I shows the numerical results for various values of $\lambda$ of the skin friction coefficient $f^{\prime \prime}(0)$ when $M=A=0.1, \operatorname{Pr}=E c=1.0$ and $S=0$ by [5] and the present results which show an excellent agreement.

TABLE I. Values of the skin friction coefficient and the Nusselt number for different values of $\lambda$ when $M=A=0.1$,

$$
E c=\operatorname{Pr}=1.0 \text { and } S=0 .
$$

\begin{tabular}{lllcc}
\hline \hline$\lambda$ & $\begin{array}{c}\text { Soid et al. } \\
(2017) \\
f^{\prime \prime}(0)\end{array}$ & Present Study & $\begin{array}{c}\text { Soid et al. } \\
(2017) \\
-\theta^{\prime}(0)\end{array}$ & $\begin{array}{c}\text { Present Study } \\
\end{array}$ \\
\hline & & & & $-\theta^{\prime}(0)$ \\
1 & 0 & 0 & 1.64764430 & 1.64764430 \\
0.5 & 0.74229441 & 0.74229441 & 1.23082763 & 1.23082763 \\
0 & 1.29973375 & 1.29973375 & 0.52573430 & 0.52573430 \\
-0.5 & 1.62053894 & 1.62053894 & -0.41366075 & -0.41366075 \\
-1.4 & 0.69681352 & 0.69681352 & -5.15777141 & -5.15777141 \\
-1.401 & 0.67060921 & 0.67060921 & -5.43163891 & -5.43163891 \\
-1.4017 & 0.63385487 & 0.63385487 & -5.88601576 & -5.88601576 \\
-1.40174 & 0.62672941 & 0.62672941 & -5.98573932 & -5.98573932 \\
\hline \hline
\end{tabular}


a)

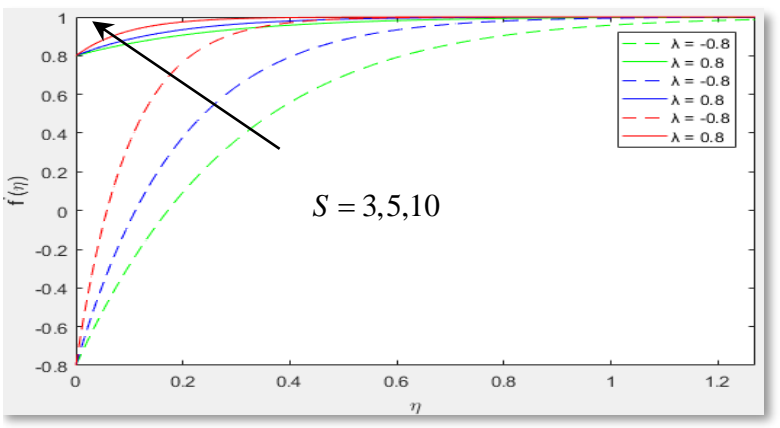

b)

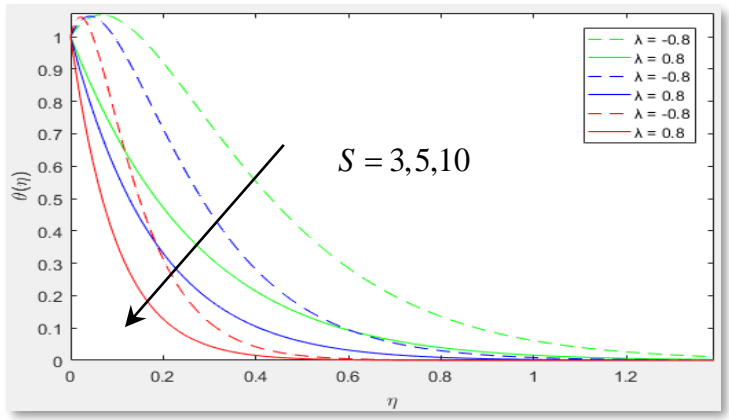

FIG. 1. Effect of different values of suction $S$ on a) velocity profile and b) temperature profile for different values of $S$ when $A=0.1, M=0.05, \operatorname{Pr}=E c=1.0$ and $\lambda=-0.8$ and 0.8 .

TABLE II.Values of the skin friction coefficient and the Nusselt number for different values of $S$ when $A=0.1, M=0.05, \operatorname{Pr}=E c=1.0$ and $\lambda=-0.8$ and 0.8 .

\begin{tabular}{llll}
\hline \hline$\lambda$ & $S$ & $f^{\prime \prime}(0)$ & $S=3,5,10$ \\
\hline-0.8 & 3.0 & 6.02969960 & -2.24731794 \\
& 5.0 & 9.42510785 & -3.35921389 \\
& 10.0 & 18.22765339 & -6.33789565 \\
\hline 0.8 & 3.0 & 0.74723224 & 3.65289992 \\
& 5.0 & 1.10234398 & 5.39572077 \\
& 10.0 & 2.05592475 & 10.07098343 \\
\hline \hline
\end{tabular}

FIG.1a) shows the velocity profile $f^{\prime}(\eta)$ for both shrinking/stretching cases. The velocity rises with the increase in $S$ for both shrinking/stretching cases and as consequence, the thickness of the boundary layer decreases. The velocity gradient increases in positive slope as the suction parameter $S$ increases for both shrinking/stretching cases. This is because suction improves the flow near the surface of the wall. Due to the positive increases of the skin friction coefficient, $f^{\prime \prime}(0)$ with the value of $S$ increases for both cases of permeable sheet, it indicates that the drag force exerts more from the fluid to the shrinking surface compared to the stretching surface as shown in TABLE II . FIG.1b) shows that the temperature at the particular point decreases with the increases of $S$ for both shrinking/stretching cases and consequently thickness of the boundary layer also decreases. The temperature slope on the surface increases in positive slope as $S$ increases for shrinking case and vice versa for stretching case where the slope of the temperature on the surface decreases in negative slope near the boundary layer. This indicates the heat transfers from the fluid to the surface for the shrinking case, while the heat transfers from the surface to the fluid for the stretching case.

a)

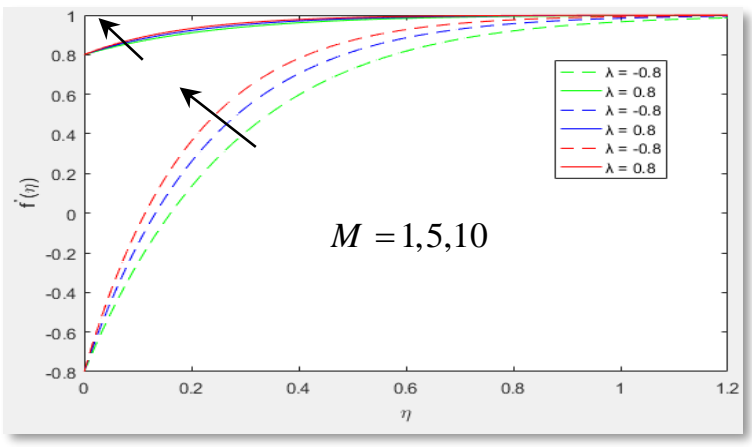

b)

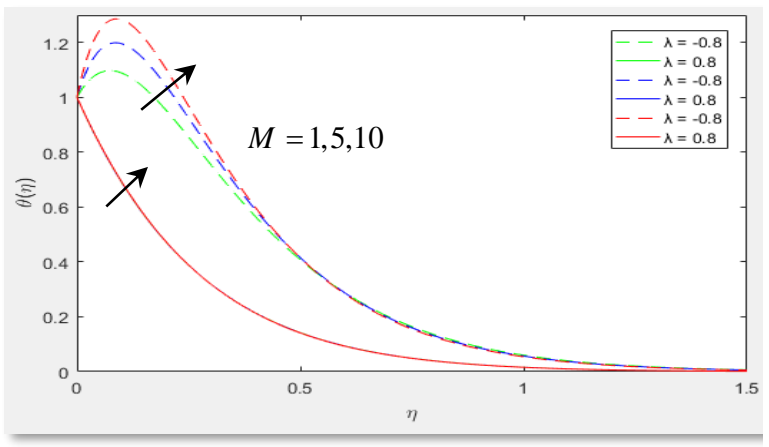


FIG. 2. Effect of various values of magnetic parameter $M$ on a) velocity profile and b) temperature profile when $A=0.1, S=3.0, \operatorname{Pr}=E c=1.0$ and $\lambda=-0.8$ and 0.8 .

TABLE III. Values of the skin friction coefficient and the Nusselt number for various values of $M$ when $A=0.1, S=3.0$, $\operatorname{Pr}=E c=1.0$ and $\lambda=-0.8$ and 0.8 .

\begin{tabular}{llll}
\hline \hline$\lambda$ & $M$ & $f^{\prime \prime}(0)$ & $-\theta^{\prime}(0)$ \\
\hline-0.8 & 1.0 & 6.44954831 & -2.97375761 \\
& 5.0 & 7.87367184 & -5.44320419 \\
& 10.0 & 9.24342592 & -7.82748409 \\
0.8 & 1.0 & 0.78682178 & 3.64631729 \\
& 5.0 & 0.92795756 & 3.62247751 \\
& 10.0 & 1.06980680 & 3.59798643 \\
\hline \hline
\end{tabular}

FIG.2a) shows that the velocity increases with the increase in $M$ for the shrinking case but slightly increases for stretching case and as consequent to it, the thickness of boundary layer decreases for both cases. Due to the positive increases of the skin friction coefficient, $f^{\prime \prime}(0)$ with the value of $M$ increases in TABLE III for both shrinking/stretching cases, it indicates that the drag force exerts from the fluid to the surface. FIG.2b) shows that the temperature at the particular point increases then gradually decreases with the increases of $M$ for the shrinking case but increases for the stretching case and consequently slightly increases the thickness of the boundary layer for both cases. The temperature slope increases in positive slope as the magnetic parameter $M$ increases in shrinking case and vice versa for the stretching case decreases in negative slope slightly near the boundary layer which give similar interpretation as the explanation in TABLE II.

It is observed in FIG.3a) that for both shrinking/stretching cases, the velocity increases with the increases in $A$ and consequently, the boundary layer is thicker for the shrinking case as compared to the boundary layer for the stretching case. The drag force exerts from the fluid to the surface due to the positive increases of the skin friction coefficient, $f^{\prime \prime}(0)$ for both cases of shrinking/stretching sheet with the increasing value of $A$ as shown in TABLE IV. FIG.3b) illustrates that the temperature at the particular point decreases with the increases of $A$ for both of the shrinking/stretching cases and consequently the thickness of the boundary layer also decreases.

a)

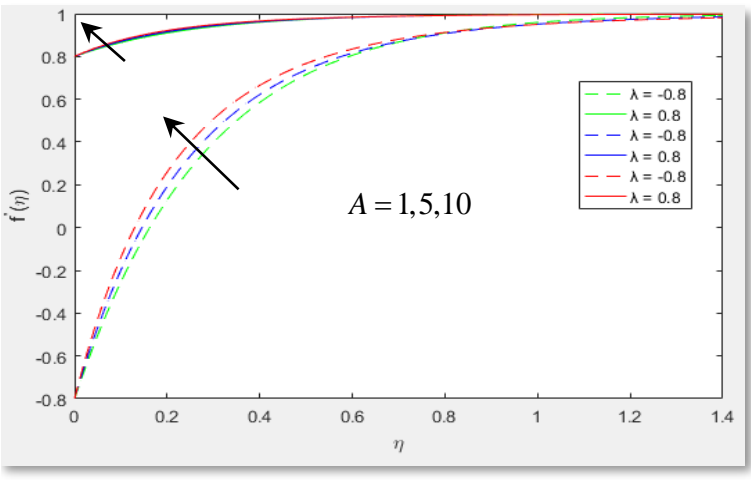

b)

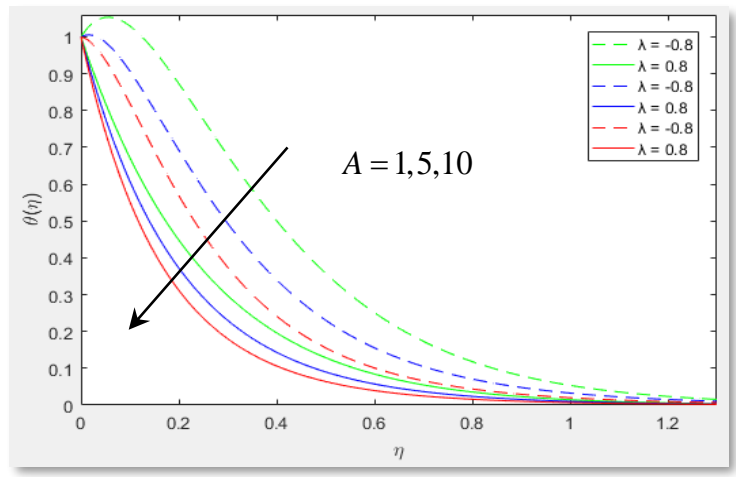

FIG. 3. Effect of various values of unsteadiness parameter $A$ on a) velocity profile and $b$ ) temperature profile when $M=0.5, S=3.0, \operatorname{Pr}=E c=1.0$ and $\lambda=-0.8$ and 0.8 . 
TABLE IV. Values of the skin friction coefficient and the Nusselt number for various values of $A$ when $M=0.5$,

\begin{tabular}{llll}
\multicolumn{4}{c}{$\operatorname{Pr}=E c=1.0, S=3.0$ and $\lambda=-0.8$ and 0.8 . } \\
\hline \hline$\lambda$ & $M$ & $f^{\prime \prime}(0)$ & $-\theta^{\prime}(0)$ \\
\hline-0.8 & 1.0 & 6.45929556 & -2.11560467 \\
& 5.0 & 7.40879038 & -0.71898715 \\
& 10.0 & 8.46588616 & 0.24662312 \\
\hline 0.8 & 1.0 & 0.78805463 & 3.94425220 \\
& 5.0 & 0.88117342 & 5.01694625 \\
& 10.0 & 0.98829569 & 6.04798365 \\
\hline \hline
\end{tabular}

Due to the heat transfers from the fluid to the surface for shrinking case causes the negative increases of the Nusselt number $-\theta^{\prime}(0)$ while for the stretching case, the heat transfers from the surface to the fluid causes the positive increases of the Nusselt number $-\theta^{\prime}(0)$. However, there is an isolated case for shrinking at $A=10.0$ as the heat transfer from the surface to the fluid.

\section{CONCLUSION}

The two dimensional heat transfer and MHD flow over a permeable stretching/shrinking sheet with ohmic heating is studied in this paper. The effect of suction parameter, unsteady parameter and magnetic parameter in porous plate are discussed. The results show that the velocity profile and skin friction coefficient increases when suction parameter increases. Furthermore, the increase in magnetic and unsteady parameters resulted in the increase in the velocity profile and the skin friction coefficient respectively.

\section{ACKNOWLEDGMENTS}

The financial supports awarded from the Universiti Teknologi MARA under the Lestari Fund (Project code: 600- IRMI/DANA 5/3/LESTARI (0140/2016)) is greatly acknowledged. The authors wish to express their sincere gratitude to the reviewers for the valuable feedbacks.

\section{REFERENCES}

[1] Mukhopadhyay, S., "MHD boundary layer flow and heat transfer over an exponentially stretching sheet embedded in a thermally stratified medium," Alexandria Engineering Journal 52, 259-265 (2013).

[2] Gelfand, I. M. \& Retakh, V. S., The Gelfand Mathematic Seminars, 1996-1999 (Springer Science \& Business Media, 2000) p.37.

[3] Vajravelu, K. \& Mukhopadhyay, S. Fluid Flow, Heat and Mass Transfer at Bodies of Different Shapes: Numerical Solutions (Academic Press, 2015) p.7-73.

[4] Farid, M. M., Mathematical Modelling of Food Processing: Contemporary Food Engineering (University of Auckland: CRC Press, 2010) p.659-687.

[5] Soid, S. K., Ishak, A. \& Pop, I., "Unsteady MHD flow and heat transfer over a shrinking sheet with ohmic heating," Chinese Journal of Physics 55(4), 1626-1636 (2017).

[6] Fadi, M. \& Shuli, L., “A comprehensive review on applications of ohmic heating (OH)," Renewable and Sustainable Energy Reviews 39, 262 -269 (2014).

[7] Rajaiah, M., Sudhakaraiah, A., Venkatalakshmi, P. \& Sivaiah, M., "Unsteady MHD free convective fluid flow past a vertical porous plate with ohmic heating in the presence of suction or injection," International Journal of Mathematics and Computer Research 2, 428-455 (2014).

[8] Sman, R.G.M.V.D, "Model for electrical conductivity of muscle meat during Ohmic heating," Journal of Food Engineering 208, 37-47 (2017). 
[9] Furtado, G.D.F., Pereira, R.N.C., Vicente, A.A. \& Cunha, R.L., "Gold gel-like emulsions of lactoferrin subjected to ohmic heating," Food Research International 103, 371-379 (2018).

[10] Santosh, C., Sawai, S. \& Susheela, C., "MHD flow and heat generation near stagnation point towards a stretching sheet in porous medium," Applied Mathematical Sciences 9, 369-378 (2015). 